\title{
Gland Puncture-Its Value in the Diagnosis of Leprosy
}

H. v. R. Mostert.

Because of the difficulty in maculo-anaesthetic leprosy of demonstrating bacilli either in scrapings from skin lesions or in smears from the nose, it was decided to test the value of smears obtained by gland puncture in some of these cases. Blood examination had previously proved of no avail (cf. Leprosy Review, Vol. VII, No. 1, p. 6).

80 native male patients with palpable glands, until recently classified as maculo-anaesthetic cases of leprosy, were chosen. Gland puncture was done prior to any examination for early nodular retrogression, although signs of an erythematous rash in 7 of these case suggested this possibility. 


\section{Technique.}

A few drops of saline in a small syringe were injected into a suitable gland. The gland was then massaged with the needle still in place. The fluid was thereafter withdrawn from the gland by suction, smeared on a slide, stained with Ziehl-Neelsen and examined for Mycobacterium leprae.

\section{Result.}

Of the 80 cases, positive gland smears were found in 13 of these cases.

On further clinical examination of the 13 positive cases all were found to be early cases of nodular leprosy. 8 showed very early signs of nodular infiltration of the face, characterized by a slight thickening and bronzing of the skin. In 6 of the 8 cases there was further evidence of an erythematous rash of the face, trunk and extremities. One other case showed signs of an erythematous rash only. Of the remaining 4 cases one had active macular lesions on the body and 3 showed no signs of activity at all on superficial examination.

In the 5 cases mentioned in whom there was no apparent evidence of nodular infiltration, subsequent scrapings from the skin of the face proved positive. Gland smears therefore served as a useful guide in enabling one to diagnose these early cases of nodular retrogression.

No evidence of nodular leprosy was found after further clinical and bacteriological investigation of the 67 maculoanaesthetic cases with negative gland smears.

The following table gives a summary of bacteriological findings in the 13 positive gland smear cases:-

$\begin{array}{lcccrr}\text { Gland smears } & \ldots & 0 & +5 \text { (3 scanty) } & ++ & +++ \\ \text { Skin Scrapings } & \ldots & 0 & 7 \text { (3 scanty) } & 6 & 0 \\ \text { Nasal Smears } & \ldots & 4 & 9 \text { (2 scanty) } & 0 & 0 \\ \text { Thick Blood Smears } & 6 & 7 \text { (all scanty) } & 0 & 0\end{array}$

Bacilli were easily demonstrated from both gland and skin. In our investigation only one series of smears was required from either source. On the other hand positive nasal and blood smear results were only obtained after repeated examinations, bacilli in thick blood smears being especially hard to find. At a somewhat more advanced stage of nodular leprosy, however, bacilli are more easily found in the blood and positive blood smears are the rule (cf. Leprosy Review, Vol VII, No. 1, page 7).

The following cases warrant special mention in that they 
tend to demonstrate that smears from the nose need not necessarily be positive in the early stages of nodular retrogression:

Case 4792 Native Male.

An old nerve case with active macules on the body, now tending to become nodular. There is an erythematous rash on the face, trunk and extremities and a few areas of early bronze coloured infiltration of the face. There is marked depilation of eyebrows.

Gland Smear (Left Inguinal) +++

Skin Smear (Right Cheek) ++

Nasal Smear - (taken monthly over a period of 6 months).

Blood Smear -

Case 6031 Native Male.

A maculo-anaesthetic case which is becoming nodular. There is an erythematous rash on the trunk and extremities, although no evidence as yet of infiltration of the face on superficial examination.

Gland Smear (Right Inguinal) + scanty.

Skin Smear (Right Cheek) + scanty.

Nasal Smear - (taken monthly over a period of 6 months).

Blood Smear + scanty.

Case 5882 Native Male.

An old nerve case with no signs of activity on superficial examination.

Gland Smear (Right Epitrochlear) ++

Skin Smear (Left Eyebrow) +

Nasal Smear - (taken monthly over a period of 6 months).

Blood Smear -

Case 6171 Native Male.

A few cutaneous macules on body. No evidence of nodular leprosy on superficial examination.

Gland Smear (Left Femoral) +

Skin Smear (Left Cheek) + scanty.

Nasal Smear -

Blood Smear -

\section{CONCLUSIONS.}

1. Gland puncture is of little or no value in the diagnosis of maculo-anaesthetic leprosy.

2. Positive gland smears are amongst the earliest indications of nodular leprosy, and are to be preferred to either nasal smears or thick blood smears in the diagnosis of these cases in the early stages. An erythematous rash on the body and infiltration of the skin are also early signs.

3. In apparently closed cases (cf. Case 5882 above), positive smears obtained by gland puncture serve as a guide to early nodular retrogression. Gland puncture is therefore of value as a test of arrest. 